\title{
Spectral efficient IR-UWB communication design for low complexity transceivers
}

\author{
Vijaya Yajnanarayana ${ }^{1 *}$, Satyam Dwivedi ${ }^{1}$, Alessio De Angelis ${ }^{2}$ and Peter Händel ${ }^{1}$
}

\begin{abstract}
Ultra wideband (UWB) radio for communication has several challenges. From the physical layer perspective, a signaling technique should be optimally designed to work in synergy with the underneath hardware to achieve maximum performance. In this paper, we propose a variant of pulse position modulation (PPM) for physical layer signaling, which can achieve raw bitrate in excess of $150 \mathrm{Mbps}$ on a low complexity in-house developed impulse radio UWB platform. The signaling system is optimized to maximize bitrate under practical constraints of low complexity hardware and regulatory bodies. We propose a detector and derive its theoretical performance bounds and compare the performance in simulation in terms of symbol error rates (SER). Modifications to the signaling, which can increase the range by 4 times with a slight increase in hardware complexity, is proposed. Detectors for this modification and a comparative study of the performance of the proposed UWB physical layer signaling schemes in terms of symbol error rates are discussed.
\end{abstract}

Keywords: Pulse position modulation; Sensor networks; Time-to-digital converter; Ultra wideband; UWB communication

\section{Introduction}

The radio technologies for communication systems generally employ a non-overlapping radio frequency (RF) spectrum. That is, every radio technology like GSM, 3G, Bluetooth, etc. uses a distinct RF spectrum. There are several radio technologies, and several new ones are emerging; as a result, RF spectrum is becoming more premium and more scarce. Communication systems using ultra wideband (UWB) offer a promising solution which can coexist with other radio technologies. This coexistence also saves expensive spectrum licensing fees $[1,2]$. The Federal Communications Commission (FCC) adopted licensefree UWB operation in the United States of America [3]. This has resulted in $7.5 \mathrm{GHz}$ of spectrum available for UWB systems. One of the direct consequences of this large bandwidth is the ability to achieve very high data rates, as given by the Shannon-Hartley theorem. Wide bandwidth also enables innovative system design such as trading data rate to avoid costly channel estimation techniques in [4] or designing the analog transmit and receive

\footnotetext{
*Correspondence: vpy@kth.se

1 Department of Signal Processing, KTH Royal Institute of Technology, Fack, 10044 Stockholm, Sweden

Full list of author information is available at the end of the article
}

structure with non-idealities in [5]. In general, there is a wide scope of data rate, range, and other parameters that can be traded off based on the application [6-8].

There are several ways in which a signal can be spread to large bandwidths. The most popular methods include frequency hopping (FH) [9], orthogonal frequency-division multiplexing (OFDM) [10], direct-sequence spread spectrum (DS-SS) [11], and time-hopping impulse radio (TH-IR) [12]. UWB based on OFDM and TH-IR have gone in to IEEE 802.15.3a and IEEE 802.15.4a standards. TH-IR schemes are most popular as they provide better performance and complexity trade-offs [7].

The use of impulse signaling (TH-IR) was proposed by Win and Scholtz in the 1990s. Their work published in [12-14] contributed significantly toward the adaptation of TH-IR for UWB. High bandwidth enables the UWB transceiver to generate narrow impulse signals; this fine time resolution can yield accurate position localization and ranging. This has enabled the application of UWB for high-precision ranging and localization. Our objective is to utilize the same platform for both communication and localization. Figure 1 shows a graphical depiction of an inhouse developed IR-UWB platform for ranging and communication. It uses a low-cost pulse generator to generate

\section{Springer}

(c) 2014 Yajnanarayana et al: licensee Springer. This is an Open Access article distributed under the terms of the Creative Commons Attribution License (http://creativecommons.org/licenses/by/4.0), which permits unrestricted use, distribution, and reproduction in any medium, provided the original work is properly credited. 
(a)

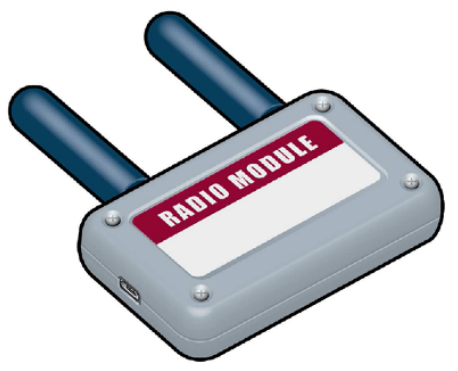

Radio module (b)

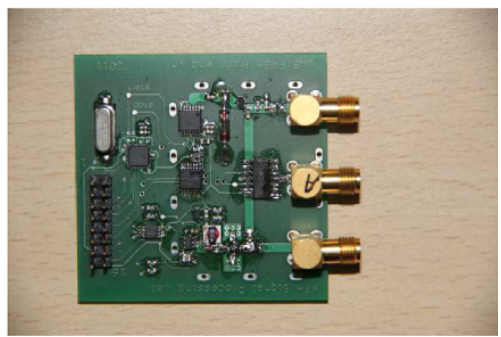

Printed circuit board (PCB)

Figure 1 Iconic model of the in-house developed impulse radio UWB-platform of size $\mathbf{6} \times \mathbf{4} \mathbf{c m}$. Iconic model of the in-house developed impulse radio UWB-platform of size $6 \times 4 \mathrm{~cm}$ for ranging and communication working in the 6-GHz regime with separate RX and TX antennas from Greenwave Scientific (details available at [19]).

sub-nanosecond pulses using step recovery diode (SRD), as described in [15]. The characterization and modeling of the UWB platform for a distance measurement system can be found in [16,17]. A detailed architectural description and experimental ranging result from a prototype of the platform have been published in [18]. The power and range of the transceiver can be easily traded by controlling the amplitude, duty cycle, and number of pulses per bit of transmission.

There are several commercial companies which develop IR-UWB products, including [20-24]. Companies like DecaWave and BeSpoon develop 802.15.4a standard specific IR-UWB products $[23,25]$. The physical layer signals of these UWB radios are defined by the standard. There are some companies like Time Domain and Ubisense which develop non-standard or custom-made communication and localization solutions [26,27]. In these UWB radios, the physical layer signaling does not adhere to any standards. The work proposed in this paper considers a methodology to maximize the communication rate through custom physical layer signaling, subject to hardware, and regulatory constraints.

The main motivation for the work is from the requirement that many UWB applications need to perform localization and communication using the same radio module $[6,28]$. The UWB radios of Time Domain and Ubisense both have localization and communication capabilities; however, these radios have minimal communication capabilities of few Kbps and physical layer signaling in them is not made public. This paper is also motivated by the fact that extensive research can be found on the design of hardware platforms and algorithms for localization and communication strategies in $[6,18,29]$, However, how to optimize the physical layer signaling for communication in view of constraints from cost-effective hardware and regulatory bodies is not a well studied problem. The achievable bitrate for the proposed methods in this paper depends on the hardware parameters of the UWB platform. The proposed methods suggest that the in-house developed UWB radio shown in Figure 1 can achieve bitrates up to $150 \mathrm{Mbps}$. The in-house UWB platform uses pulse round-trip time (RTT) for localization. It has a range of about $10 \mathrm{~m}$ with an accuracy of $30 \mathrm{~cm}$ in practical scenarios. It has a digital processing section based on a field-programmable gate array (FPGA), which interfaces with analog UWB sections to generate required analog pulsed waveforms for transceiver operation. The modulator and demodulator algorithms proposed in this paper can be programmed in FPGA, for processing UWB communication signals. This paper proposes two signaling schemes with one requiring higher complexity in modulation and demodulation, however can increase the range by nearly 4 times without compromising on the bitrate. This is believed to have an interest in its own right, as it corresponds to (or outperforms) today's state of the art. Although the work considers the in-house developed UWB radio (Figure 1) for demonstrating the techniques, the results are of general importance which can enable engineers to follow similar methodology to exploit the hardware and spectrum to achieve a higher possible range and bitrate.

In this context, through this paper we propose a method for communication using low-cost and low complexity hardware architecture which can perform ranging, localization, and communication. The main contributions of this paper are summarized below:

- A method to design a custom physical layer signaling to maximize the data rate by optimal choice of modulation parameters, given the constraints from hardware and regulatory bodies, is illustrated. An algorithm for such a modulator with no memory between symbols called a no-memory modulator is proposed. 
- A spectrally more efficient modulator, which can improve the range of the communication by introducing the memory between symbols called with-memory modulator, is proposed. This has a marginal increase in complexity of modulator and demodulator algorithms with an increase in range by 4 times.

- A no-memory modulator is analyzed by deriving an expression for the symbol error rate (SER) performance. A detector algorithm for the no-memory modulator is proposed, and its SER performance is verified through simulation.

- The detector algorithm for the with-memory modulator is proposed and detector performance of no-memory and with-memory signaling are compared in simulation.

This paper is organized as follows: In Section 2, we discuss the system model and pulse shapes used in the impulse radio. Section 3 discusses UWB constraints. Section 4 is on low complexity UWB hardware platform for localization and communication, and Section 5 details the design of physical layer signal construction and modulator algorithms. Section 6 describes detectors and their performance in terms of symbol error rates. Finally, Section 7 details the conclusions from the design and results demonstrated.

\section{Pulse construction and system model}

A wide range of pulse shapes have been explored for UWB communication from rectangular to Gaussian [30]. Gaussian pulses and their derivatives, usually called monopulses, are effective due to the ease of construction and good resolution in both time and frequency. In many cost-effective hardware designs, these shapes are generated without any dedicated circuits. A simple transistor or diode, which is turned 'on' and 'off' to generate a narrow rectangular pulse, will form an approximate Gaussian shape due to the imperfections in micro-electronic design [31].

The time domain Gaussian pulse with mean $\mu$ and variance $\sigma^{2}$ can be written as

$$
\frac{1}{2 \pi \sigma^{2}} e^{\frac{-(t-\mu)^{2}}{2 \sigma^{2}}}
$$

A more useful form of this equation for system design is defined in [31]. This is a scaled version of (1) with zero mean and variance $\tau^{2} / 4 \pi$. This is given by

$$
s(t)=-e^{-2 \pi\left(\frac{t}{\tau}\right)^{2}}
$$

In a typical UWB device, when the signal passes through the UWB antenna, it will have a differentiation effect on the signal. A similar effect is observed when the receiver receives the pulses. The first- and second-order Gaussian pulses are given by

$$
\begin{aligned}
& s^{\prime}(t)=\frac{d s(t)}{d t}=\frac{4 \pi t}{\tau^{2}} e^{\frac{-2 \pi t^{2}}{\tau^{2}}} \\
& s^{\prime \prime}(t)=\frac{d^{2} s(t)}{d t^{2}}=-4 \pi e^{\frac{-2 \pi t^{2}}{\tau^{2}}}\left(\frac{-\tau^{2}+4 \pi t^{2}}{\tau^{4}}\right)
\end{aligned}
$$

For analytical and simulation analysis, we have used the power normalized second-order Gaussian pulse as shown in (5).

$$
p^{2}(t)=\frac{\left(s^{\prime \prime}(t)\right)^{2}}{\int_{-\infty}^{\infty}\left(s^{\prime \prime}(t)\right)^{2} d t}
$$

Several modulation techniques are proposed in the literature using narrow pulses [12,32]. Primarily, they are variants of pulse position modulation (PPM), binary phase shift keying (BPSK) or on-off keying (OOK). Since our objective is to employ low complexity hardware structure to perform synchronized ranging and communication, a variant of PPM-based signaling for communication is used. This can reuse the hardware structure (having round trip time (RTT) calculation logic for ranging) for detection and demodulation of physical layer signal. This is further illustrated in Section 4.

The system model comprises of three parts; transmitter, channel, and receiver, as shown in Figure 2. The transmitter generates the PPM variant signal. This is a modified pulse specified in [12] with 1 symbol per pulse and no time hopping. The transmitted output signal is given by

$$
W_{\mathrm{tr}}(t)=\sqrt{E_{p}} \sum_{n} p\left(t-n T_{\mathrm{S}}-d_{n} \Delta-\gamma_{n}\right)
$$

where $T_{\mathrm{S}}$ is the symbol period, $d_{n}$ is the $n$-th symbol value $d_{n} \in[0, . ., M-1], p(t)$ is the normalized pulse such that $\int_{-\infty}^{\infty} p^{2}(t) d t=1, E_{p}$ is the energy of the pulse, $\gamma_{n}$

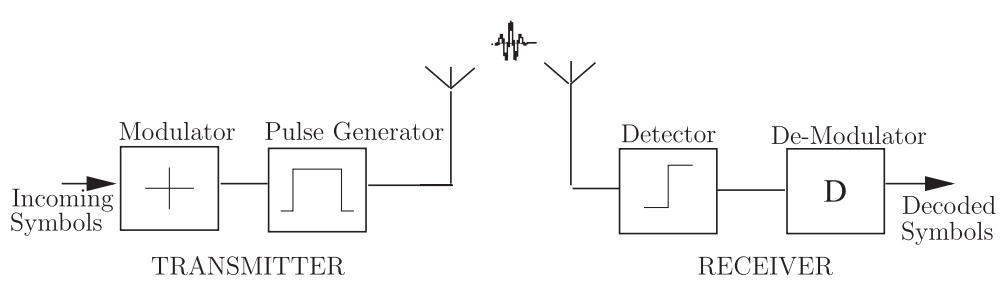

Figure 2 System model consisting transmitter, channel, and receiver. 
is a parameter coming from the constraints of the typical UWB hardware which will be explained later, $\Delta$ is the modulation index, and $\log _{2} M$ is the modulation order. Though in our model each symbol is defined by one pulse, it can be easily extended to multiple pulses for each symbol so that communication rate and detectability can be traded.

In UWB applications which use ranging for localization, the detectors rely on the first arriving path or line of sight (LOS); this is in contrast to traditional channel measurement and modeling. However, for extremely short distance high-speed communication with highly directional antennas, we can adopt a deterministic single path channel [8]. These short distance high-speed UWB applications include transferjet and wireless USB (wUSB). The message information is embedded in the time axis of PPM signals. Due to the high time resolution of UWB signals, the performance of the system is sensitive to the timing jitter and synchronization [33,34]. The effect of channel, imperfect timing/synchronization, and receiver front end thermal noise will cause errors while demodulating the PPM signals. We combine the effect of channel, imperfect timing/synchronization, together with receiver front end to introduce a random jitter (shift on time axis) along with noise $n(t)$ as shown in (7). For analytical and simulation purposes, we assume Gaussian distribution with zero mean and variance $\sigma_{j}^{2}$ for jitter [35]. Thus, the received signal is given by

$$
W_{\mathrm{rx}}(t)=\sqrt{E_{r}} \sum_{n} p\left(t-n T_{\mathrm{S}}-d_{n} \Delta-\gamma_{n}-j(n)\right)+n(t)
$$

where $E_{r}$ is the energy of the received pulse, $j(n) \sim$ $\mathcal{N}\left(0, \sigma_{j}^{2}\right)$ is the random jitter in the received signal, and $n(t)$ is the receiver noise on the received signal.

The third part of the system model is the detector/demodulator, which demodulates the signal represented in (7). In the signal model, a single-user UWB system with no multiple access interference is assumed. However, it is straightforward to extend the techniques proposed in this paper for multi-user system.

In the subsequent sections, we will show how to optimally design the modulator and detector to the constraints of hardware and regulatory bodies. We will also evaluate the theoretical performance of the demodulator for the chosen modulator. In the next section, we will discuss some of the challenges in transmission and detection of UWB pulses from the regulatory bodies and hardware perspective.

\section{UWB constraints}

The FCC regulation is one of the most popular regulations for UWB, and most of the regulations in other countries are derived from this regulation. The FCC regulations, [3], define a UWB system as any intentional radiator having an absolute 10-dB bandwidth greater than $500 \mathrm{MHz}$ or a relative bandwidth greater than $20 \%$. Since UWB systems have to co-exist with other narrowband technologies, the compliance requirements from regulatory bodies for UWB systems are very stringent to ensure that they do not interfere with the existing narrowband systems. This makes the design of UWB for communication challenging. These requirements are generally specified through constraints on maximal average power $P_{\text {av }}$ and maximal peak power $P_{\mathrm{pk}}$. The average power, $P_{\mathrm{av}}$, is measured using a spectrum analyzer (SA) with resolution bandwidth $(\mathrm{RBW}) B_{\mathrm{av}}=1 \mathrm{MHz}$. Maximal average power constraints are specified through spectral masks. Figure 3 shows the FCC mandated spectral mask for indoor UWB emissions. $P_{\mathrm{pk}}$ should not exceed $0 \mathrm{dBm}$ when measured using an SA with RBW set to $50 \mathrm{MHz}$.

The pulse repetition rate (PRF) (that is, $1 / T_{\mathrm{S}}$ in (6)) of the IR-UWB signal plays a significant role on how the UWB device impacts other narrowband receivers in its range; these receivers are called victim receivers [3]. If the PRF is larger than the bandwidth of the victim receiver, then the emission may appear as noise like to the victim receiver. This effect is proportional to the average power of the UWB signal within the receiver's bandwidth. If pulse rate is smaller than the victim receiver's bandwidth, then UWB signal would appear like impulse noise to the victim receiver and the effect is proportional to the peak power of the UWB signal. Thus, at low PRF the output levels are constrained by the limit on peak emission levels and at high PRF by the limit on average emission levels [3].

In [36], the authors specify the existence of two distinct regimes where only one of the two power constraints are active for the IR-UWB signal. For PRF less than $1 \mathrm{MHz}$, peak constraints are active; for PRF greater than $1 \mathrm{MHz}$, average power constraints are active. The signaling proposed in this paper is optimized for high-rate data communication and hence, high PRF is assumed as $\gg 1 \mathrm{MHz}$ yielding only the average power constraint relevant.

\section{UWB hardware}

Larger bandwidth of ultra wideband signals also enables suboptimal receiver designs, which are more efficient from cost and complexity perspective. Some of these lowcost designs can be found at $[18,37]$. In this correspondence, we propose the hardware architecture platform of [18] for communication. The modulator algorithm on FPGA will generate control signals required to trigger the step recovery diode to generate UWB pulses. On the receive side, the transceiver has an energy detector; whenever the signal energy crosses crosses a certain threshold, it sends a 'Start/Stop' signal to time-to-digital converter (TDC). TDC measures the interval between the pulses, 


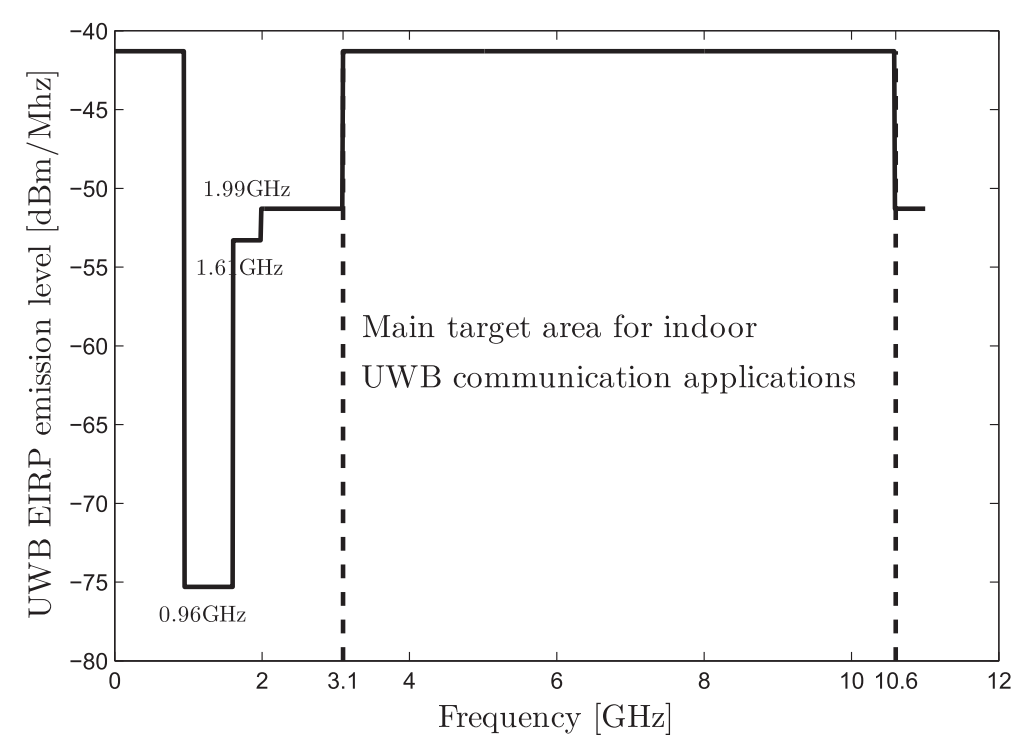

Figure 3 Spectral mask specified by FCC showing the equivalent isotropic radiated power (EIRP) versus frequency for indoor UWB system.

which carries information in the PPM variant physical layer signaling. This information is further processed by the demodulator algorithm on FPGA to demodulate the symbol. Estimating the round-trip time on unmodulated signal can be used to localize objects as discussed in [18]. Thus, this low complexity transceiver can be used for both localization and communication [38].

In general, in a low complexity UWB transmitter, it is not possible to transmit arbitrary close pulses because of the recovery time required for the micro-electronic devices used in them. This creates a constraint on the signaling that the pulses need to be separated by at least a minimum distance equal to the recovery time. This is the reason for having the $\gamma_{n}$ in (6). Also, at the detector it is not possible to resolve between arbitrarily close pulses. Thus, the modulation index $\Delta$ in (6) cannot be arbitrarily small.

\section{Modulator}

A periodic train of impulses will result in a spectral comb formation in the power spectral density (PSD). This results in an inefficient usage of power and reduces the range of UWB nodes as the spectral peaks can cross the power levels specified in Figure 3. One way to overcome the spectral comb formation is to randomize the pulse interval. As an illustrative example, we can choose the probability density function (PDF) of pulse repetition period $T_{\mathrm{PRT}}$ as a uniform distribution given by

$$
\mathrm{p}\left(T_{\mathrm{PRT}}\right)= \begin{cases}\frac{1}{2 T_{\mathrm{S}}-T} & \text { If } T \leq T_{\mathrm{PRT}} \leq 2 T_{\mathrm{S}} . \\ 0 & \text { Otherwise. }\end{cases}
$$

Here $T$, which is $\ll T_{\mathrm{S}}$ is the pulse width of the pulses. $T_{\mathrm{PRT}}$ is varied from $T$ instead of 0 to avoid collision between pulses. The train of these pulses is shown in Figure $4 \mathrm{a}$ and its power spectrum is shown in Figure $4 \mathrm{~b}$. For this kind of signaling on average, the pulse rate will be close to $1 / T_{\mathrm{S}}$ and the power spectrum will be smooth, as shown in Figure $4 \mathrm{~b}\left(T_{\mathrm{S}}=18 \mathrm{~ns}, T=1 \mathrm{~ns}\right)$.

As discussed in Section 4, it is not possible from the hardware perspective to generate arbitrarily close pulses. Also, from the detector perspective it is not possible to resolve the timing between arbitrarily close pulses. For example, in the hardware architecture proposed in [18], the step recovery diode used in the transmitter has a fixed recovery time, preventing the generation of close pulses; and TDC used in the detector has a fixed time resolution, preventing the detection of close pulses. One way to design the signaling is to have $\gamma_{n}$ in (6) equal to the minimum separation needed between the pulses $T_{\mathrm{ms}}$, and modulation index $\Delta$ in (6) equal to the detectors sensitivity (TDC time resolution). Thus, the resulting transmitted signal assuming 1 symbol per pulse is given by

$$
W_{\mathrm{tr}}(t)=\sum_{n} p\left(t-n T_{\mathrm{S}}-d_{n} \Delta-T_{\mathrm{ms}}\right)
$$

Figure 5 shows the illustration of this signaling. Each symbol has a fixed gap of $T_{\mathrm{ms}}$ in the beginning between $\left[0, T_{\mathrm{ms}}\right]$ and the modulated RF pulse in the interval $M \Delta$ between $\left(T_{\mathrm{ms}}, T_{\mathrm{S}}\right.$ ]. If $\log _{2} M$ is the modulation order, then the symbol time $T_{\mathrm{S}}$ and bitrate $R_{b}$ is given by

$$
T_{\mathrm{S}}=T_{\mathrm{ms}}+\Delta M
$$

and

$$
R_{b}=\frac{\log _{2} M}{T_{\mathrm{S}}} .
$$


(a)

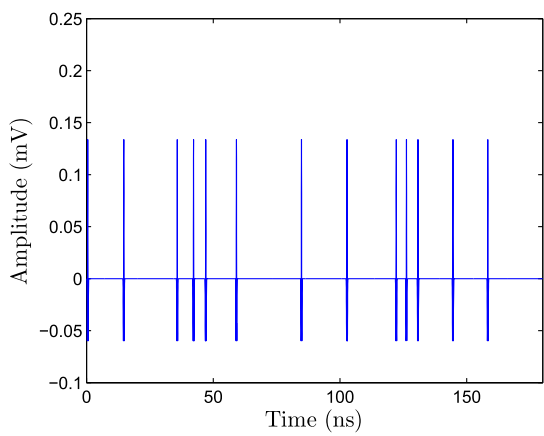

Train of randomized pulses (b)

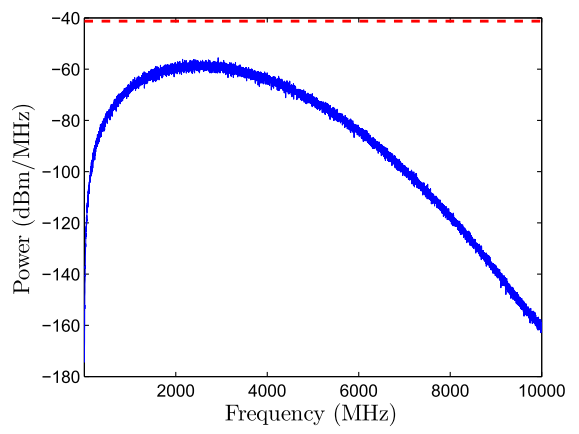

Power spectral density (PSD) of randomized pulses.

Figure 4 Train of pulses with randomized pulse interval and its PSD. (a) $T_{\mathrm{S}}=18 \mathrm{~ns}$ is employed and 1,000 pulses are considered for computing PSD. Dashed line in (b) indicates $-41.3 \mathrm{dBm} / \mathrm{MHz}$.

For any UWB hardware, the $T_{\mathrm{ms}}$ and $\Delta$ are fixed; they come from the two UWB hardware constraints discussed above. The modulation parameter $M$ can be picked to maximize the bitrate. To do this, (11) is evaluated and the optimal $M$ that maximizes the bitrate $R_{b}$ is chosen. The variation of $R_{b}$ versus $M$ for various ratio's of $T_{\mathrm{ms}} / \Delta$ (indicating different transceiver hardware) is shown in Figure 6a. The bitrate $R_{b}$ for the optimal choice of $M$ versus $T_{\mathrm{ms}} / \Delta$ is shown in Figure 6b.

The bitrate peak to around $160 \mathrm{Mbps}$ at $M=8$ when the typical parameter values of $T_{\mathrm{ms}}=10 \mathrm{~ns}$ and $\Delta=1 \mathrm{~ns}$ for the in-house UWB platform is considered.

The modulator algorithm which modulates the input symbol vector, $\mathbf{d}$, is shown in Algorithm 1 . The algorithm is initialized with $X$ and $Y$, denoting the TDC resolution and diode recovery time $[18,39]$. The method generateRFPulse in line 6 of the algorithm is used to generate the train of RF pulses as shown in (9). In the in-house prototype UWB platform, this is accomplished by generating a trigger control signal from FPGA to the step recovery diode in transmitter [18]. Since no memory is employed between symbols in this signaling, it is called no-memory signaling. This modulator was introduced and briefly described in [38]. A variant of the M-PPM signal shown in (9) with $T_{\mathrm{ms}}=10 \mathrm{~ns}$ and modulation index $\Delta=1 \mathrm{~ns}$ is generated. Five pulses of this are shown in Figure 7a. Each symbol duration, $T_{\mathrm{S}}=18 \mathrm{~ns}$, resulting in PRF $\gg 1 \mathrm{MHz}$ causing only average power constraint being active. Each symbol constitutes a fixed constant gap of $T_{\mathrm{ms}}=10 \mathrm{~ns}$, and 3 bits of information are modulated in the remaining $M \Delta=8$ ns (since $\Delta=1 \mathrm{~ns}, M=8$ ). We cannot achieve a smooth PSD as in Figure 4b because now, pulse train has deterministic gaps between pulses $\left(T_{\mathrm{ms}}\right)$ and the pulse positions are quantized $(\Delta)$ for it to work with the chosen UWB hardware; however, the data can be assumed to be random because of interleaving and randomization in the symbol rate chain of the physical layer; this does smoothen the PSD. The PSD of this signaling scheme is shown in Figure $7 \mathrm{~b}$.

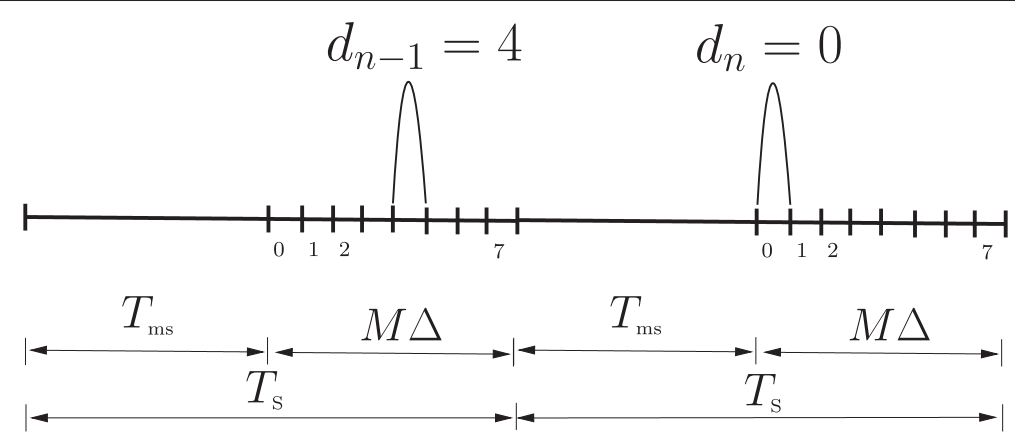

Figure 5 Signaling employed to ensure minimum separation needed between pulses. Signal employed to have $\gamma_{n}$ in (6) equal to the minimum separation needed between the pulses $T_{\mathrm{ms}}$ and modulation index $\Delta$ in (6) is equal to the detectors sensitivity. Here $M=8$ is assumed. No memory is employed in the signaling between symbols, hence it is called no-memory signaling. 
(a)

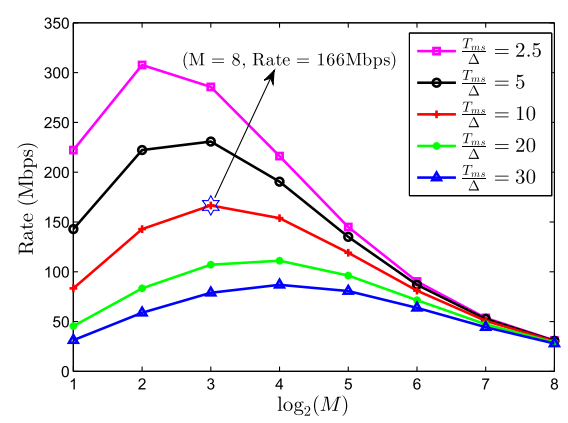

Bitrate $\left(R_{b}\right)$ versus modulation order $(M)$ (b)

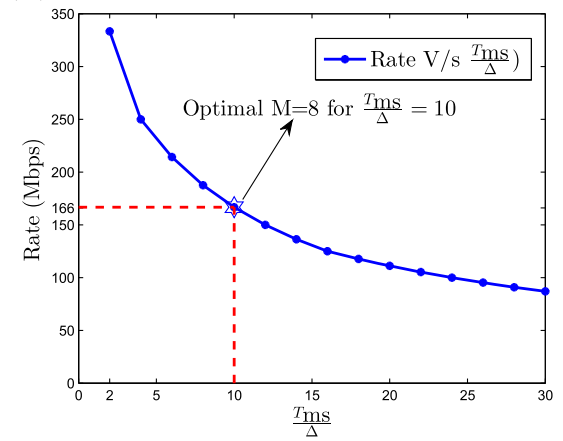

Rate versus $T_{\mathrm{ms}} / \Delta$ for the choice of optimal $M$.

Figure 6 Variation of bitrate $R_{\boldsymbol{b}}$ with parameters $\boldsymbol{M}$ and $T_{\mathrm{ms}} / \boldsymbol{\Delta}$. Choice of optimal $M$, where bitrate peaks depends on $T_{\mathrm{ms}} / \Delta$. Bitrate peaks at $M=8$ for the nominal parameter values for the in-house hardware consisting of $T_{\mathrm{ms}}=10 \mathrm{~ns}$ and $\Delta=1 \mathrm{~ns}$.

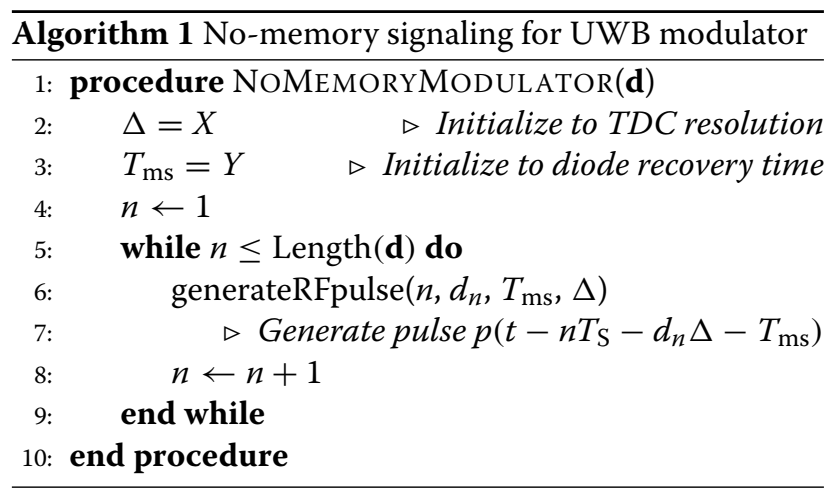

The signaling employed above has a fixed gap, $T_{\mathrm{ms}}$ in every symbol, as given in (9). This ensures that minimum separation between pulses is greater than or equal to the recovery time of the diode. If the modulator structure is altered to remember the position of the transmitted pulse for the previous symbol, then the minimum gap needed for the current symbol can be reduced. We define this reduced gap as $T_{\mathrm{ms}}^{\prime}$ and it is illustrated in Figure 8. In Figure 8 , gap $T_{\mathrm{ms}}^{\prime}$ is reduced by $3 \Delta$ by remembering the position of the pulse in the previous symbol. The total separation between the pulses is $T_{\mathrm{ms}}=T_{\mathrm{ms}}^{\prime}+3 \Delta$. Since the duration of the symbol is constant, the reduction in the gap, $T_{\mathrm{ms}}^{\prime}$ will result in an increased region for modulated RF pulses; this increases the time interval bin widths (modulation index), thereby increasing the detectability. Figure 8 shows the increase in bin widths for the second symbol $\left(d_{n}=0\right)$ as gap, $T_{\mathrm{ms}}^{\prime}$ reduced by $3 \Delta$. Since in this method the transmitter needs to remember the past transmitted symbol to decide the $T_{\mathrm{ms}}^{\prime}$ for the current symbol, we call this signaling as with-memory signaling. The algorithm for implementing this modulator is shown in Algorithm 2. It is initialized with $X$ and $Y$, denoting the (a)

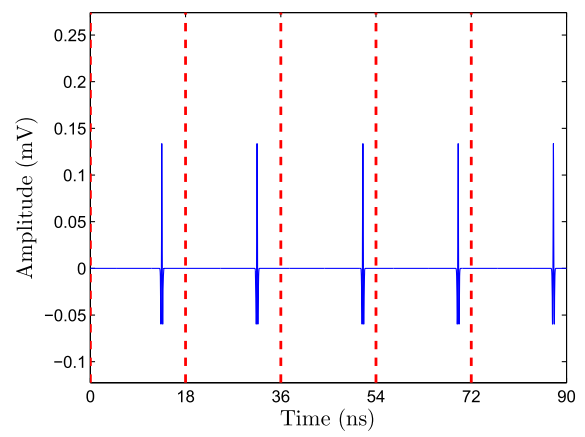

No-memory signaling waveforms. (b)

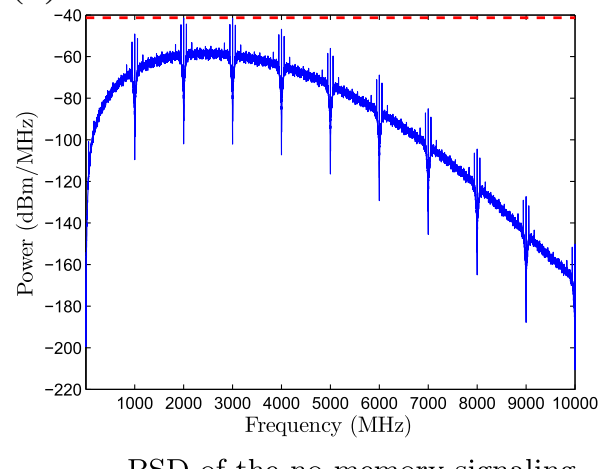

PSD of the no-memory signaling.

Figure 7 No-memory signaling and its PSD with $\boldsymbol{M}=\mathbf{8}, \boldsymbol{\Delta}=\mathbf{1} \mathbf{n s}$, and $\boldsymbol{T}_{\mathrm{ms}} / \boldsymbol{\Delta}=\mathbf{1 0}$. For computing PSD, 1,000 pulses are considered. The dashed vertical line in (a) indicates the symbol boundary, and the dashed horizontal line in (b) indicates $-41.3 \mathrm{dBm} / \mathrm{MHz}$, average power constraint defined by the FCC for in-house UWB radio operating at $6 \mathrm{GHz}$. 
TDC resolution, $\Delta$, and diode recovery time, $T_{\mathrm{ms}}$. The algorithm takes a vector of symbols, $\mathbf{d}$, and calls generateRFPulse with different bin widths $\Delta_{n}$ and gap $T_{\mathrm{ms}}^{\prime}$ to generate the train of RF pulses defined in (9).

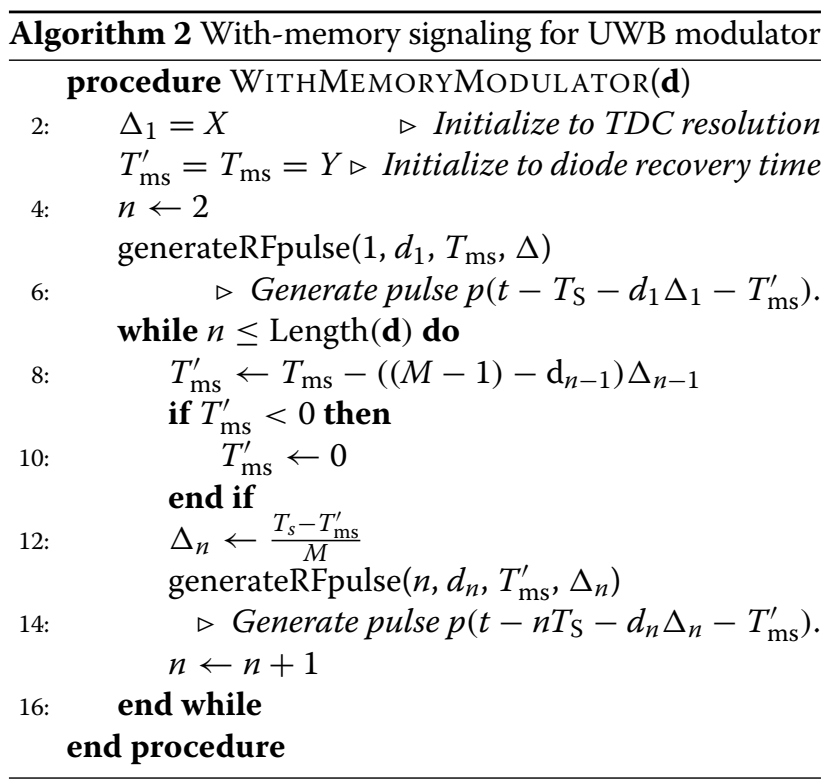

The two primary benefits of this signaling are summarized below.

1. There is better detectability due to an increase in the bin widths (modulation index) as explained before.

Later in the paper, we compare the performance (in terms of symbol error rate (SER)) of this signaling with no-memory signaling.

2. There is better randomization of the pulses. In contrast to no-memory signaling, there are no deterministic gaps and pulses spread to all regions of the symbol interval.
The impact of 2) is further smoothing of the PSD; Figure 9a shows five symbols in the time domain, and Figure 9b shows the PSD with the modified signaling of randomly generated 1,000 bits. The order $M$ is chosen as 8 , recovery time $T_{\mathrm{ms}}=10 \mathrm{~ns}$, and modulation index $\Delta=1 \mathrm{~ns}$ as discussed above.

Comparing Figure $9 \mathrm{~b}$ and Figure $7 \mathrm{~b}$ indicates that withmemory signaling will smoothen the PSD more than the no-memory signaling. This will enable the transmitter to generate pulses at a higher amplitude without violating the mask specification of the regulatory bodies, thereby increasing the range. Comparing Figure $9 \mathrm{a}$ and Figure 7a indicates that the pulse amplitude can be increased by approximately 4 times compared to the no-memory signaling, without violating the regulatory body specifications and with the same hardware constraints. For the deterministic single-path propagation model, the path loss, PL, is given by

$$
\mathrm{PL}=\frac{(4 \pi d f)^{2}}{c}
$$

where $f$ is the frequency of operation, $d$ is the range, and $c$ is the speed of light [40]. The received power, $P_{\text {rx }}$, for transmitted power, $P_{\mathrm{tx}}$, is given by (13).

$$
P_{\mathrm{rx}}=\frac{P_{\mathrm{tx}}}{\mathrm{PL}}
$$

An amplitude increase of 4 times results in 16 times $(12 \mathrm{~dB})$ increase in power. Since path loss is proportional to the square of the distance as given by (12), for any given received power and frequency of operation, if $d_{1}$ and $d_{2}$ are ranges for transmitters employing no-memory and with-memory signaling, then

$$
\frac{P_{\mathrm{tx}}}{d_{1}^{2}}=\frac{16 P_{\mathrm{tx}}}{d_{2}^{2}}
$$

which leads to

$$
d_{2}=4 d_{1}
$$

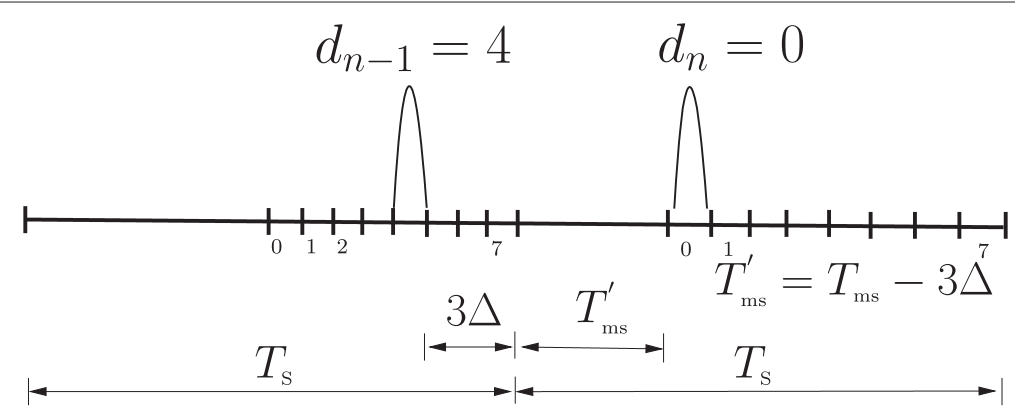

Figure 8 With-memory signaling for UWB modulator. With-memory signaling for UWB modulator showing the reduction in the gap $T_{\mathrm{ms}}^{\prime}$ in the current symbol by remembering the position of the pulse in the previous symbol. $M=8$ is assumed. 
(a)

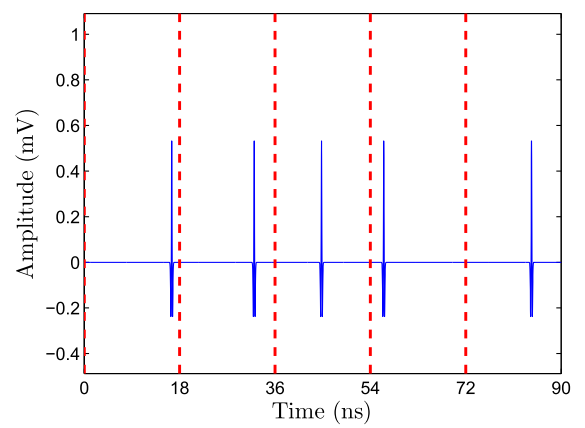

With-memory signaling waveforms. (b)

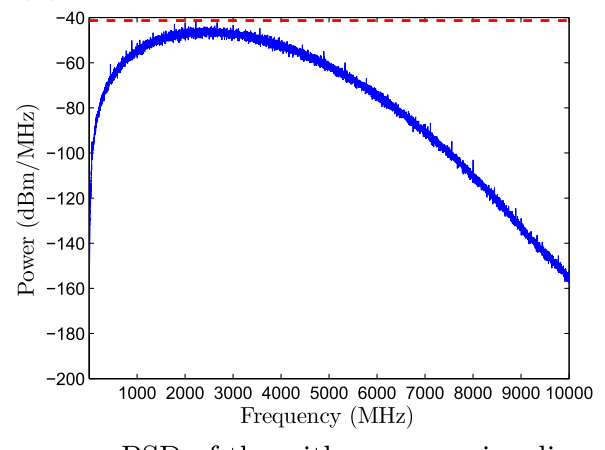

PSD of the with-memory signaling

Figure 9 With-memory signaling and its PSD with $\boldsymbol{M}=\mathbf{8}, \boldsymbol{\Delta}=\mathbf{1} \mathrm{ns}$ and $\boldsymbol{T}_{\mathrm{ms}} / \boldsymbol{\Delta}=\mathbf{1 0}$. For computing PSD, 1,000 pulses are considered. The dashed vertical line in (a) indicates the symbol boundary, and the dashed horizontal line in (b) indicates $-41.3 \mathrm{dBm} / \mathrm{MHz}$, average power constraint defined by the FCC for in-house UWB radio operating at $6 \mathrm{GHz}$.

Eq. 15 means that the range can be increased by 4 times compared to the no-memory signaling. This increased range comes with a cost of increased complexity in the modulator, as shown in Algorithm 2.

In the next section, we will evaluate the performance of the demodulator for the proposed signaling scheme.

\section{Detector performance}

In this section, we propose a detector for the modulators proposed before and evaluate its performance. For analytical discussion, we assume that the symbol timing acquisition procedure has been performed prior to data transmission. For the no-memory signaling modulator with $M=8$, a hard decision demodulator is designed. The demodulator uses the quantized 8 time interval bins as defined in Table 1. This is also illustrated in Figure 10.

The intervals for two corner bins are larger because the detector can exploit the dead time $T_{\mathrm{ms}}$ left for the diode recovery. The algorithm for the hard decision demodulation is shown in Algorithm 3. The algorithm demodulates the received vector, $\mathbf{r}$, into a vector DemodulatedSymbol. The PeakPosition in line 3 of the algorithm returns the peak position of the received pulse.

The performance bounds for the no-memory signaling are derived in terms of SER. The received signal is as defined in (7). For no-memory signaling transmitter, the

Table 1 Time intervals used for hard decision demodulation

\begin{tabular}{lc}
\hline & Quantized time intervals for hard decision demodulation \\
\hline$\nabla_{0}$ & {$\left[\left(\frac{T_{\mathrm{ms}}}{2}\right)-\left(\frac{T_{\mathrm{ms}}}{2}+\Delta\right)\right]$} \\
\hline$\nabla_{i}$ & {$[(i \Delta-((i+1) \Delta)]$ for $i \in[1, . .6]$} \\
\hline$\nabla_{7}$ & {$\left[\left(\frac{T_{\mathrm{ms}}}{2}+6 \Delta\right)-\left(T_{\mathrm{S}}+\frac{T_{\mathrm{ms}}}{2}\right)\right]$} \\
\hline
\end{tabular}

received signal at the receiver will have $\gamma_{n}=T_{\mathrm{ms}}$ and is given by

$$
W_{\mathrm{rx}}(t)=\sqrt{E_{r}} \sum_{n} p\left(t-n T_{\mathrm{S}}-d_{n} \Delta-T_{\mathrm{ms}}-j(n)\right)+n(t)
$$

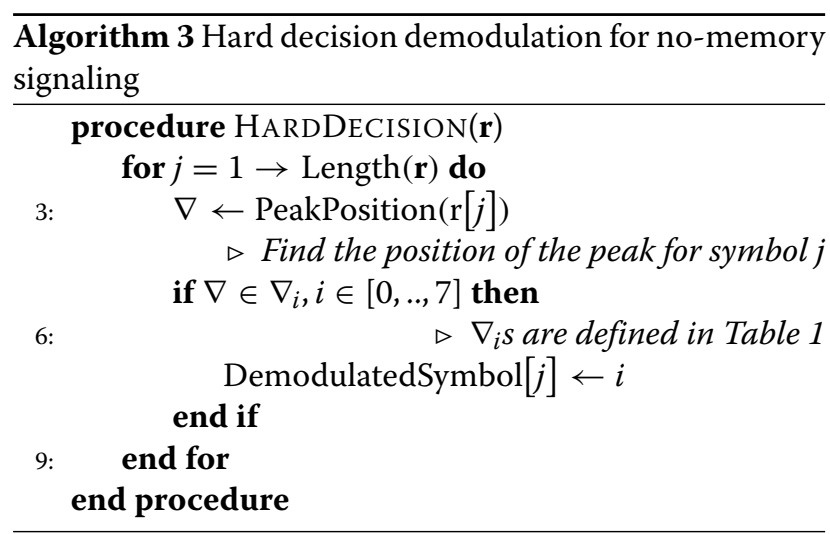

As shown in Table 1, from the detector perspective, there are three different types of symbols. The first symbol 0 and the last symbol 7 have a much larger detection probability, since their decision interval $\left(\nabla_{0}, \nabla_{7}\right)$ is large. The middle symbols $[1-6]$ have the same and smaller decision intervals $\nabla_{i}=\Delta, i \in[1,6]$. In general, if the modulation order is $M$, and all symbols are equally likely, then the corner symbols will occur each with a probability of $1 / M$ and middle symbols with $(M-2) / M$. If $P_{e 1}$ is the probability of a symbol error for the middle symbol, and $P_{e 2}$ and $P_{e 3}$ are the probabilities of symbol errors for the corner symbols, then the probability of symbol error $P_{e}$ is the average of the three types of errors; $P_{e 1}, P_{e 2}$, and $P_{e 3}$. 


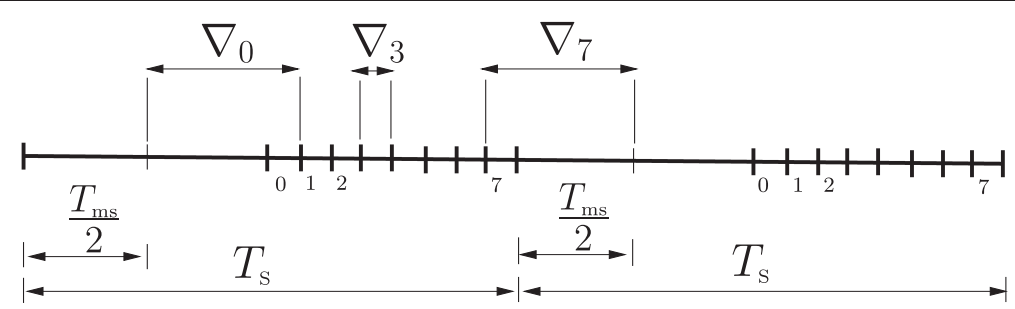

Figure 10 Quantized time bins used by the hard decision detector. The corner bins are wider than the middle bins.

Refer Table 1 and Figure 10.

$$
\begin{aligned}
P_{e} & =\left(\frac{M-2}{M}\right) P_{e 1}+\left(\frac{1}{M}\right) P_{e 2}+\left(\frac{1}{M}\right) P_{e 3} \\
& =\left(\frac{M-2}{M}\right) P_{e 1}+\left(\frac{2}{M}\right) P_{e 2}
\end{aligned}
$$

where we assumed $P_{e 2}=P_{e 3}$ in the second equality. Further,

$$
\begin{aligned}
P_{e}= & 2\left(\frac{M-2}{M}\right) \operatorname{Pr}\left(x>\frac{\Delta}{2}\right) \\
& +\frac{2}{M}\left(\operatorname{Pr}\left(x>\frac{\Delta}{2}\right)+\operatorname{Pr}\left(x>\frac{\Delta+T_{\mathrm{ms}}}{2}\right)\right) \\
= & 2\left(\frac{M-1}{M}\right) \operatorname{Pr}\left(x>\frac{\Delta}{2}\right) \\
& +\frac{2}{M} \operatorname{Pr}\left(x>\frac{\Delta+T_{\mathrm{ms}}}{2}\right)
\end{aligned}
$$

When the signal referred in (16) is passed through a peak detector, the combined effect of the jitter $j(n)$ and noise $n(t)$ on the peak detector will cause an error in the peak position. This error in the peak position $w(n)$ can be assumed to be Gaussian distributed with mean 0 and variance $\sigma^{2}$, Therefore, we get

$$
\begin{aligned}
\operatorname{Pr}\left(x>\frac{\Delta}{2}\right) & =\frac{1}{\sqrt{2 \pi \sigma^{2}}} \int_{\frac{\Delta}{2}}^{\infty} \exp \left(\frac{-x^{2}}{2 \sigma^{2}}\right) d x \\
& =\mathrm{Q}\left(\frac{\beta}{2}\right)
\end{aligned}
$$

Q-function is the tail probability of the standard normal distribution. The parameter $\beta$ is the ratio of bin width $\Delta$ to standard deviation $\sigma$ of the random errors in the peak position. Similarly,

$$
\begin{aligned}
\operatorname{Pr}\left(x>\frac{\Delta+T_{\mathrm{ms}}}{2}\right) & =\frac{1}{\sqrt{2 \pi \sigma^{2}}} \int_{\frac{\Delta+T_{\mathrm{ms}}}{2}}^{\infty} \exp \left(\frac{-x^{2}}{2 \sigma^{2}}\right) d x \\
& =\mathrm{Q}\left(\frac{\Delta+T_{\mathrm{ms}}}{2 \sigma}\right) \\
& =\mathrm{Q}\left(\frac{(1+\alpha) \beta}{2}\right)
\end{aligned}
$$

Here in the third equality, transceiver specific ratio $\left(T_{\mathrm{ms}} / \Delta\right)=\alpha$ is assumed. Substituting (18) and (19) in (17), the symbol error probability $P_{e}$ as given by

$$
P_{e}=2\left\{\left(\frac{M-1}{M}\right) \mathrm{Q}\left(\frac{\beta}{2}\right)+\frac{1}{M} \mathrm{Q}\left(\frac{(1+\alpha) \beta}{2}\right)\right\}
$$

where $\beta=\Delta / \sigma$ and $\alpha=T_{\mathrm{ms}} / \Delta$.

The performance is evaluated in simulations with $M=8, \Delta=1 \mathrm{~ns}$, and $T_{\mathrm{ms}}=10 \mathrm{~ns}(\alpha=10)$. The simulation consisted of of 200,000 randomly generated symbols. The symbol error rate for various $\beta$ values are shown in Figure 11. A strong correlation for $P_{e}$ between theory and simulation is observed.

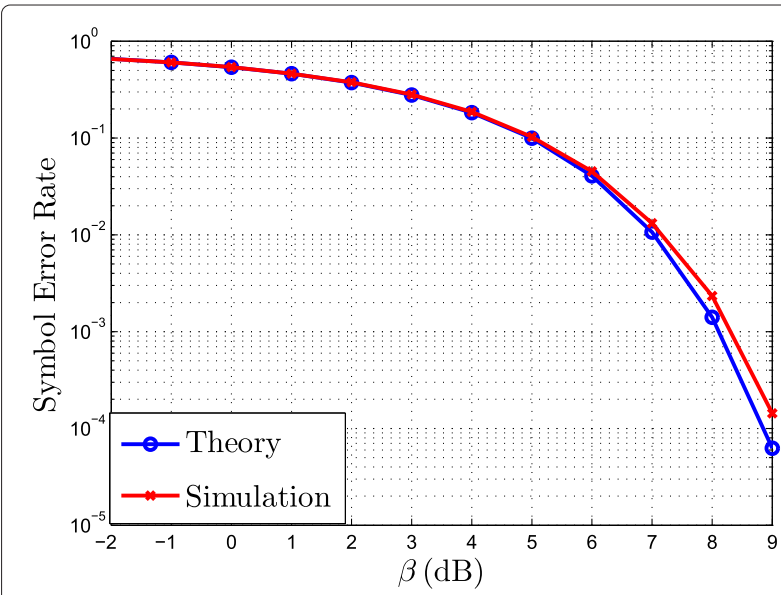

Figure 11 Comparison of theoretical and simulation performance. Comparison of theoretical and simulation performance in terms of symbol error rates for hard decision detection-based demodulator for no-memory signaling. 
Earlier, we proposed a modified signaling with memory in the modulator (with-memory signaling), which claimed to have two benefits.

1. A better detectability due to an increase in the bin widths (modulation index), as explained before.

2. Better randomization of the pulses. Unlike in the no-memory signaling, there are no deterministic gaps and pulses are spread to all regions of the symbol interval.

The impact of 2) is further smoothing of the PSD, which is demonstrated earlier with the PSD plots. To quantify the performance improvement due to 1 ), detector performance of with-memory signaling and no-memory signaling needs to be compared. We implemented a hard decision detection algorithm for the with-memory signaling. This algorithm is described in Algorithm 4. The algorithm demodulates the received vector, $\mathbf{r}$, into a vector DemodulatedSymbol. Parameters $T_{\mathrm{ms}}$ and $T_{\mathrm{S}}$ in the algorithm should be the same as those used in the with-memory modulator algorithm defined in Algorithm 2. The PeakPosition in line 4 of the algorithm returns the peak position of the received pulse. This algorithm is similar to the no-memory demodulator defined in Algorithm 3, except that the bin width $\Delta_{i}$ changes between symbols, and it depends on the previous bin width and previously decoded symbol.
It is not straightforward to obtain a closed-form analytical expression for the performance of with-memory signaling. In with-memory signaling on average $T_{\mathrm{ms}}^{\prime}<$ $T_{\mathrm{ms}}$, if we assume that all the symbols are equally likely, then the average symbol value $(\in[0, M-1])$ for the previous symbol is $(M-1) / 2$; therefore, on average $T_{\mathrm{ms}}^{\prime}$ is given by

$$
T_{\mathrm{ms}}^{\prime}=T_{\mathrm{ms}}-\frac{(M-1)}{2} \Delta
$$

The average bin width for with-memory signaling is given by

$$
\begin{aligned}
\Delta^{\prime} & =\frac{T_{\mathrm{S}}-T_{\mathrm{ms}}^{\prime}}{M} \\
& =\frac{T_{\mathrm{ms}}-T_{\mathrm{ms}}^{\prime}+M \Delta}{M}
\end{aligned}
$$

where we in the second equality used (10). Further applying (21) to (23), we get

$$
\Delta^{\prime}=\left(\frac{3 M-1}{2 M}\right) \Delta
$$

If we define $\beta^{\prime}$ as the ratio of $\Delta^{\prime} / \sigma$, then we have

$$
\beta^{\prime}=\left(\frac{3 M-1}{2 M}\right) \beta
$$

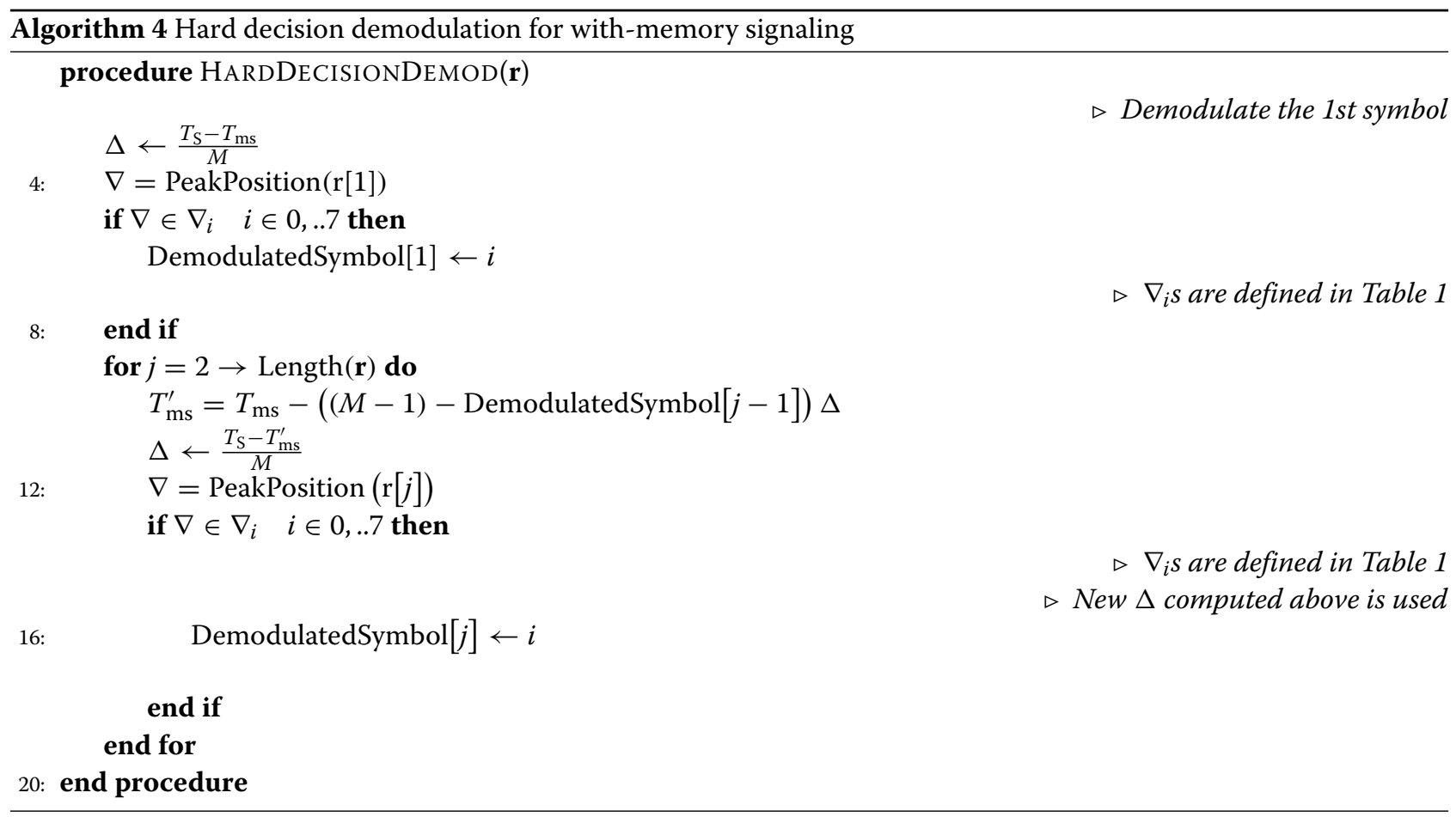


Substituting (25) in (20), we get the symbol error probability for the with-memory signaling and is given by (26).

$$
\begin{aligned}
P_{e}= & 2\left\{\left(\frac{M-1}{M}\right) \mathrm{Q}\left(\frac{(3 M-1) \beta}{4 M}\right)\right. \\
& \left.+\frac{1}{M} \mathrm{Q}\left(\frac{(1+\alpha)(3 M-1) \beta}{4 M}\right)\right\}
\end{aligned}
$$

From (24), for $M=8$ we get an average bin width for the $\Delta^{\prime}=1.43 \Delta$, This increase in the bin width will result in better detectability of symbols at the receiver. In simulation, it was observed that the bin width increases by $\Delta^{\prime}=1.32 \Delta$. This reduction in the bin width is due to line $9-12$ in the modulator Algorithm 2, where in order to ensure one pulse per symbol period, $T_{\mathrm{ms}}^{\prime}$ is forced to zero, resulting in the reduction of bin width for the transmitted symbol. The performance of detector for with-memory signaling is evaluated in simulation with 200,000 randomly generated samples, and the results are compared with no-memory signaling. Figure 12 compares the performance of the hard decision detectors for no-memory and with-memory signaling schemes. A gain of approximately $1 \mathrm{~dB}$ can be achieved in terms of SNR at a symbol error rate of $10^{-2}$ by using with-memory signaling.

The presented symbol error rate (SER) performance for the proposed signaling schemes are valid for the transceivers which are in line of sight (LOS) with short distance between them and having highly directional antennas as discussed in Section 2. For these systems, we can assume a simple AWGN channel model. However, for systems having fading channels with multi-path, channel equalization and time of arrival (TOA) estimation needs to be performed prior to demodulation. The SER performance of the proposed signaling schemes in such systems depends on the performance of channel equalization and TOA estimation algorithms.

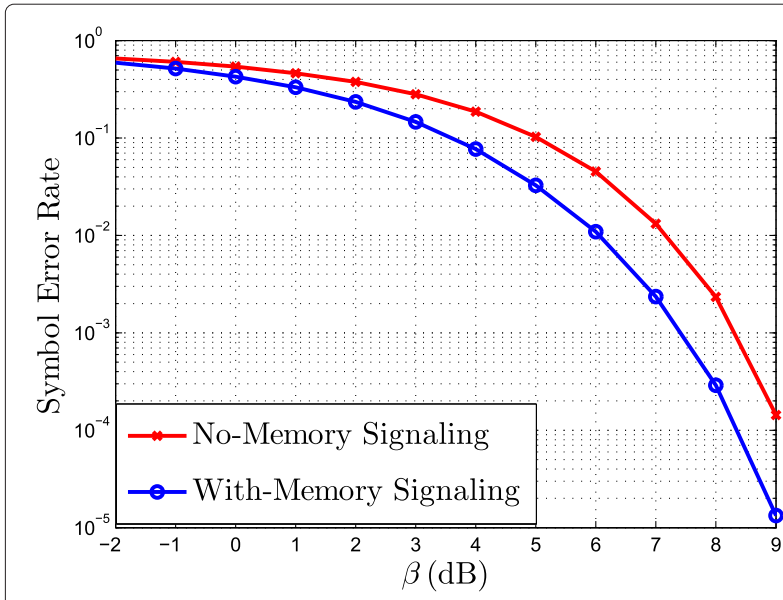

Figure 12 Comparison of performance of no-memory and with-memory signaling in terms of symbol error rate.

\section{Conclusion}

In this paper, we proposed a custom signaling which is a variant of PPM signaling for IR-UWB communication. We also proposed an alternative signaling called withmemory signaling, which requires memory in the modulator and demodulator, however, can further smoothen PSD compared to no-memory signaling. The result of this is illustrated in Figure 9b. We showed that range can be increased by 4 times compared to no-memory signaling, without violating the regulatory body constraints. This gain comes with a cost of increased complexity in the modulator and demodulator, as discussed in the modulator and demodulator Algorithms 2 and 4 for with-memory signaling. We also derived the theoretical closed-form expression for a hard decision demodulator with no-memory signaling. We compared the performance with the derived theoretical result; this is illustrated in Figure 11. We implemented a detector for the with-memory signaling proposed in this paper. The detector performance of with-memory signaling is compared with the detector performance for no-memory signaling. We showed that with-memory signaling can improve the detector performance by approximately $1 \mathrm{~dB}$ at $10^{-2} \mathrm{SER}$. Results are illustrated in Figure 12.

The performance of the proposed signaling methods of the paper are demonstrated in simulations in order to assess the performance gains without many platform dependencies. The proposed schemes are valid for any low-cost UWB hardware platform employing a step recovery diode at the transmitter and having a need for minimum time resolution between pulses at the detector for detection. The transceiver in Figure 1 will be an integral part of our next generation infrastructure free indoor position system [41], the radio here should be used not only for ranging but also for the wireless communication and thus fulfill a need for the proposed method. Today, we can use commercial UWB ranging like Time Domain for this purpose; however, these systems do not have the high bitrate communication capabilities. The results of the proposed signaling methods indicate the possibility of achieving a higher bitrate in excess of $150 \mathrm{Mbps}$ with low probability of error in detection as suggested by the performance curves, using the parameters from our transceiver hardware. With these findings, we intend to further develop the work to implement the proposed algorithms into our transceiver system and evaluate the performance of in-house transceiver hardware. The proposed algorithms and methods are explained in the context of in-house UWB hardware; however, the results are of general importance which could enable engineers to apply similar methods and algorithms toward the design of UWB communication system. 


\section{Competing interests}

The authors declare that they have no competing interests.

\section{Acknowledgements}

Parts of the work have been funded by The Swedish Agency for Innovation Systems (VINNOVA).

\section{Author details}

${ }^{1}$ Department of Signal Processing, KTH Royal Institute of Technology, Fack, 10044 Stockholm, Sweden. ${ }^{2}$ Engineering Department, University of Perugia, Perugia, Italy.

\section{Received: 25 June 2014 Accepted: 23 September 2014}

\section{Published: 3 October 2014}

\section{References}

1. MGD Benedetto, T Kaiser, AF Molisch, I Oppermann, C Politano, D Porcino, Ultra-wideband Communication Systems: A Comprehensive Overview. (Hindawi Publishing Corporation, New York, 2006)

2. H Nikookar, R Prasad, Introduction to Ultra Wideband for Wireless Communications. Signals and Communication Technology Series. (Springer, London, 2009). http://books.google.se/books?id=6ico-tJpmcYC

3. Federal Communications Commission, Revision of part 15 of the commision's rules regarding ultra-wideband transmission systems. First report and order. Technical report, FCC, Washington DC, ET Docket 98-153 (2002)

4. R Hoctor, H Tomlinson, Delay-hopped transmitted-reference RF communications, in Ultra Wideband Systems and Technologies, 2002. Digest of Papers. 2002 IEEE Conference On (2002), p. 265-269. doi:10.1109/UWBST.2002.1006368

5. AM Orndorff, Transceiver Design for Ultra-Wideband Communications, Electrical and Computer Engineering. Virginia Polytechnic Institute and State University (2004)

6. H Luecken, Communication and Localization in UWB Sensor Networks. PhD thesis, ETH ZURICH (2012)

7. J Zhang, PV Orlik, Z Sahinoglu, AF Molisch, P Kinney, UWB Systems for Wireless Sensor Networks. Proc. IEEE. 97(2), 313-331 (2009). doi:10.1109/JPROC.2008.2008786

8. AF Molisch, Ultrawideband propagation channels-theory, measurement, and modeling. IEEE Trans. Veh. Tech. 54(5), 1528-1545 (2005). doi:10.1109/TVT.2005.856194

9. L-L Yang, L Hanzo, Residue number system assisted fast frequency-hopped synchronous ultra-wideband spread-spectrum multiple-access: a design alternative to impulse radio. IEEE J. Sel. Area. Comm. 20(9), 1652-1663 (2002). doi:10.1109/JSAC.2002.805059

10. A Batra, J Balakrishnan, AG Dabak, R Gharpurey, P Fontaine, H Lin, U.S. Patent 20040151109 A1, 5 Aug 2004

11. R Fisher, R Kohno, H Ogawa, H Zhang, K Takizawa, M Mc Laughlin, M Welborn, DS-UWB Physical Layer Submission. 802.15 task group 3a. IEEE P, Los Alamitos (2005), 15-40

12. MZ Win, RA Scholtz, Ultra-wide bandwidth time-hopping spreadspectrum impulse radio for wireless multiple-access communications. IEEE Trans. Commun. 48(4), 679-689 (2000). doi:10.1109/26.843135

13. RA Scholtz, Multiple access with time-hopping impulse modulation, in Military Communications Conference, 1993. MILCOM '93. Conference Record. Communications on the Move., IEEE, vol. 2, (1993), p. 447-4502. doi:10.1109/MILCOM.1993.408628

14. MZ Win, RA Scholtz, Impulse radio: how it works. IEEE Comm. Lett. 2(2), 36-38 (1998). doi:10.1109/4234.660796

15. A De Angelis, M Dionigi, R Giglietti, P Carbone, Experimental comparison of low-cost sub-nanosecond pulse generators. IEEE Trans. Instrum. Meas. 60(1), 310-318 (2011). doi:10.1109/TIM.2010.2047591

16. A De Angelis, M Dionigi, A Moschitta, R Giglietti, P Carbone, Characterization and modeling of an experimental UWB, pulse-based distance measurement system. IEEE Trans. Instrum. Meas. 58(5), 1479-1486 (2009). doi:10.1109/TIM.2008.2009204

17. D Strömberg, A De Angelis, P Händel, A low-complexity adaptive-threshold detector for pulse UWB systems, in Int. Workshop ADC Modelling, Testing Data Converter Analysis Design/IEEE, (2011), p. 294-299
18. A De Angelis, S Dwivedi, P Handel, Characterization of a flexible UWB sensor for indoor localization. IEEE Trans. Instrum. Meas. 62(5), 905-913 (2013). doi:10.1109/TIM.2013.2243501

19. Broadband omnidirectional antenna - Elite-2460, [online]. Available: http://www.greenwavescientific.com/

20. Timedomain Company. http://www.timedomain.com/

21. Ubisense Company. http://www.ubisense.net/

22. Zebra Company. http://www.zebra.com/

23. BeSpoon Company. http://www.bespoon.com/

24. Decawave Company. http://www.decawave.com/

25. DW1000 - DecaWave's precise indoor location and communication chip, [online]. Available: http://www.decawave.com/

26. Time Domain PulsON (P400) Ranging and Communications Module (RCM). [Online] Available:. http://www.timedomain.com

27. The Series 7000 Ubisense UWB sensors.[Online]. Available:. http://www. ubisense.net/

28. J Rantakokko, P Handel, M Fredholm, F Marsten-Eklöf, User requirements for localization and tracking technology: a survey of mission-specific needs and constraints, in Indoor Positioning and Indoor Navigation (IPIN), 2010 International Conference On (2010), p. 1-9. doi:10.1109/IPIN.2010.5646765

29. S Gezici, Z Tian, GB Giannakis, H Kobayashi, AF Molisch, HV Poor, Z Sahinoglu, Localization via ultra-wideband radios: a look at positioning aspects for future sensor networks. IEEE Signal Process. Mag. 22(4), 70-84 (2005). doi:10.1109/MSP.2005.1458289

30. M Ghavami, LB Michael, S Haruyama, R Kohno, A novel UWB pulse shape modulation system. Wireless Pers. Comm. 23, 105-120 (2002). 10.1023/A:1020953424161

31. M Ghavami, LB Michael, R Kohno, Ultra Wideband Signals and Systems in Communication Engineering. (John Wiley, West Sussex, 2007)

32. K Witrisal, G Leus, G Janssen, M Pausini, F Troesch, T Zasowski, J Romme, Noncoherent ultra-wideband systems. IEEE Signal Process. Mag. 26(4), 48-66 (2009). doi:10.1109/MSP.2009.932617

33. I Guvenc, H Arslan, Performance evaluation of UWB systems in the presence of timing jitter, in Ultra Wideband Systems and Technologies, 2003 IEEE Conference On, (2003), p. 136-141. doi:10.1109/UWBST.2003.1267818

34. S Gezici, AF Molisch, HV Poor, H Kobayashi, The tradeoff between processing gains of an impulse radio UWB, system in the presence of timing jitter. IEEE Trans. Commun. 55(8), 1504-1515 (2007). doi:10.1109/TCOMM.2007.902536

35. H Wymeersch, J Lien, MZ Win, Cooperative localization in wireless networks. Proc. IEEE. 97(2), 427-450 (2009) doi:10.1109/JPROC.2008.2008853

36. F Troesch, F Althaus, A Wittneben, Pulse position precoding exploiting UWB power constraints, in Signal Processing Advances in Wireless Communications, 2005 IEEE 6th Workshop On, (2005), p. 395-399. doi:10.1109/SPAWC.2005.1506054

37. H Khani, H Nie, W Xiang, Z Xu, Z Chen, Low complexity suboptimal monobit receiver for transmitted-reference impulse radio UWB systems, in Global Communications Conference (GLOBECOM), 2012 IEEE, (2012), p. 4084-4089. doi:10.1109/GLOCOM.2012.6503756

38. V Yajnanarayana, S Dwivedi, A De Angelis, P Handel, Design of impulse radio UWB transmitter for short range communications using PPM signals, in Electronics, Computing and Communication Technologies (CONECCT), 2013 IEEE International Conference On, (2013), p. 1-4. doi:10.1109/CONECCT.2013.6469303

39. A Zhu, F Sheng, A Zhang, An implementation of step recovery diode-based UWB pulse generator, in Ultra-Wideband (ICUWB), 2010 IEEE International Conference On, vol. 2, (2010), p. 1-4. doi:10.1109/ICUWB.2010.5614726

40. M Skolnik, Radar Handbook, Third Edition. Electronics electrical engineering. (McGraw-Hill Education, New York, 2008). http://books. google.se/books?id=76uF2Xebm-gC

41. J-O Nilsson, D Zachariah, I Skog, P Händel, Cooperative localization by dual foot-mounted inertial sensors and inter-agent ranging. EURASIP J. Adv. Signal Process. 2013(164) (2013). doi:0.1186/1687-6180-2013-164

\section{doi:10.1186/1687-1499-2014-158}

Cite this article as: Yajnanarayana et al:: Spectral efficient IR-UWB communication design for low complexity transceivers. EURASIP Journal on Wireless Communications and Networking 2014 2014:158. 